Pacific Journal of Mathematics

ON LOEWI LENGTH OF RINGS 


\title{
ON LOEWY LENGTH OF RINGS
}

\author{
V. P. Camillo and K. R. Fuller
}

Associated with each ring $R$ over which every nonzero right module has a minimal submodule is an ordinal number called its right (lower) Loewy length. The concern here is with the various possible left and right Loewy lengths of such rings with zero radical and with the possible right-left symmetry of this minimal submodule condition. In particular, if $R$ has finite right Loewy length $n$ then $R$ has left Loewy length $\leqq 2^{n}-1$.

All rings are associative rings with identity. Denoting the socle of a module $M$ by Soc (M), the (lower) Loewy series for $M$ is defined transfinitely by: $S_{0}=0, S_{\alpha+1} / S_{\alpha}=\operatorname{Soc}\left(M / S_{\alpha}\right)$ and, if $\alpha$ is a limit ordinal, $S_{\alpha}=\bigcup_{\beta<\alpha} S_{\beta}$. (See Bass [1, p. 470].) If $M=S_{\alpha}$ for some ordinal number $\alpha$ then $M$ is called a (lower) Loewy module. The Loewy length of such a module is $L(M)=\gamma$, the least ordinal $\gamma$ with $M=S_{\gamma}$. We call a ring $R$ a right (resp., left) Loewy ring in case the regular representation $R_{R}$ (resp., ${ }_{R} R$ ) is a Loewy module. (Năstăsescu and Popescu [6] use the term "semi-artinian" to denote such a ring.) A ring $R$ is easily seen to be a right Loewy ring if and only if each of its right modules has a nontrivial (hence, essential) socle. Over such a ring each right module $M$ is a Loewy module of length $L(M) \leqq$ $L\left(R_{R}\right)$ and, since $R_{R}$ is finitely generated, $L\left(R_{R}\right)$ cannot be a limit ordinal.

As part of his Theorem $\mathrm{P}$, Bass [1] proved that a ring $R$ is left perfect (i.e., its (Jacobson) radical $J=J(R)$ is left $T$-nilpotent and $R / J$ is semisimple) if and only if $R$ is right Loewy and contains no infinite orthogonal set of idempotents. No doubt inspired by this result, Năstăsescu and Popescu [6] proved that a ring $R$ is right Loewy if and only if its radical $J$ is left $T$-nilpotent and $R / J$ is right Loewy. Thus we are led to study Loewy rings with zero radical. After first modifying an example of Osofsky [7] to show that there are primitive left and right Loewy rings of arbitrary infinite left and right lengths and that a primitive right Loewy ring need not be left Loewy, we prove that a right Loewy ring of finite length must also be left Loewy of finite length, but that these two lengths are neither independent nor necessarily equal. Then, recalling that the left and right Loewy series for a (von Neumann) regular ring are the same, we show that there exist both commutative and primitive regular Loewy rings of arbitrary length.

1. Right Loewy length vs. left. Osofsky [7], in answer to a 
question of Bass, proved that, given any two infinite ordinals $\varphi$ and $\gamma$, there exists a left and right perfect ring $R$ with $L\left(R_{R}\right)=\gamma+1$ and $L\left({ }_{R} R\right)=\varphi+1$; and that there exists a left perfect ring (necessarily right Loewy) with $L\left(R_{R}\right)=\gamma+1$ and $\operatorname{Soc}\left({ }_{R} R\right)=0$. In such rings $R$ all the "action" must take place in the radical (i.e., if $R$ is right perfect, $\left.L\left(R_{R}\right) \leqq L\left(J_{R}\right)+L(R / J)=L(J)+1\right)$. Indeed it was apparently heretofore not known whether a ring with zero radical could be Loewy with $L\left({ }_{R} R\right) \neq L\left(R_{R}\right)$. The following lemma allows us to build such Loewy rings with zero radical by "putting Osofsky's rings on top of" the socle of the ring of linear transformation of a vector space. Before proceeding to the lemma we recall (see [3, Chapter IV]) the ring of linear transformations of a vector space is a $P M I$ ring. That is, it is a primitive ring containing a minimal right (equivalently, left) ideal; and so its left and right socles (the transformations of finite rank) coincide, are homogeneous, and, as left and right ideals are both faithful and essential.

LEMMa 1.1. Let $B$ be an algebra over a field $K$. Then there is a PMI algebra $R$ over $K$ with $\operatorname{Soc}(R)=S$ and subalgebra $\hat{B} \cong B$ such that

$$
R=\hat{B}+S \text { and } \hat{B} \cap S=0 ;
$$

and hence $R / S \cong B$.

Proof. Let $V_{K}=B^{(N)}$ be a countable direct sum of copies of $B$, considered as a vector space over $K$. Let $T=\operatorname{End}\left(V_{K}\right)$ the ring of linear transformations of $V_{K}$, viewed as left operators. Then the mapping $b \mapsto \hat{b}$ where

$$
\hat{b}:\left(b_{1}, \cdots, b_{n}, 0, \cdots\right) \longmapsto\left(b b_{1}, \cdots, b b_{w}, 0, \cdots\right)
$$

is an injective algebra homomorphism $B \rightarrow T$ such that $\hat{b}$ has infinite rank whenever $b \neq 0$. Now let $S=\operatorname{Soc}(T)$, the transformations of $V_{K}$ of finite rank, and let

$$
R=\hat{B}+S .
$$

Then, since $S$ is an ideal in $T, R$ is a subring of $T$, and clearly $\hat{B} \cap S=0$. By the Structure Theorem for PMI rings [3, p. 75], $R$ is a $P M I$ ring with socle $S$.

Now, letting $B$ be one of the rings of Osofsky discussed above ( $B$ is an algebra), we have at once:

Proposition 1.2. Let $\phi$ and $\gamma$ be infinite ordinal numbers. Then there is a primitive left and right Loewy ring $R$ with 


$$
L\left(R_{R}\right)=\gamma+1 \text { and } L\left({ }_{R} R\right)=\varphi+1 .
$$

Moreover, there exist primitive right Loewy rings, of arbitrary infinite Loewy length, that are not left Loewy.

Of course the model for Loewy rings is the semiprimary rings, which one may characterize as left perfect rings of finite right (or equivalently, left) Loewy length. The left and right Loewy lengths of a semiprimary ring are the same-one greater than the nilpotency index of its radical. We shall now show that finite Loewy length on one side of a ring implies it on the other, but that if the ring is not semisimple modulo its radical (i.e., perfect) then the two lengths can differ.

THEOREM 1.3. If $R$ is a right Loewy ring of finite Loewy length $n$, then $R$ is a left Loewy ring of Loewy length at most $2^{n}-1$.

Proof. The proof is by induction on the right Loewy length. Let $L\left(R_{R}\right)=n, S=\operatorname{Soc}\left(R_{R}\right)$, and $N=J \cap S$, where $J$ is the Jacobson radical of $R$. Then, since $N \subset J$ and $S_{R}$ is semisimple, we have $S N=0$. Thus ${ }_{R} N$ is an $R / S$ module. Since $R / S$ has right Loewy length $n-1$, it has left Loewy length at most $2^{n-1}-1$ by inductive hypothesis. Clearly $L\left({ }_{R} R\right)$ is an upper bound for the Loewy length of any left $R$ module, so the Loewy length of ${ }_{R} N$ is less than or equal to $2^{n-1}-1$.

We claim that $S / N$ is semisimple as a left $R$ module. To see this, note that $S_{R}=\left(\Sigma \oplus e_{i} R\right) \oplus N$, where $e_{i}^{2}=e_{i}$ and the $e_{i} R$ are simple right $R$ modules. This is true because $N$ is a summand of $S_{R}$, since $S_{R}$ is semisimple; and the simple components of any complimentary summand are generated by idempotents, since they are not nilpotent. Now, the proof of [3, Proposition 1, p. 65] shows that if $e_{i} R$ is simple, and $R e_{i}$ contains no nonzero nilpotent left ideals then $R e_{i}$ is also simple. But $(R / N)\left(e_{i}+N\right)$ cannot contain any nonzero nilpotent left ideals, because, since $N^{2}=0$, any such left ideal would lift back to a nilpotent left ideal in $R e_{i}+N \subseteq S$, which would be contained in $J \cap S=N$. Thus each $\left(e_{i}+N\right)$ generates a simple left $R$ module, so $\left(R e_{i} R+N\right) / N$ is semisimple as a left $R / N$ module. But $S=\Sigma R e_{i} R+N$ so that $S / N$ is left semisimple.

Now it is clear that $L(M) \leqq L(N)+L(M / N)$ for any submodule $N \subset M$. Thus

$$
\begin{aligned}
L(R) & \leqq L(R / S)+L(S / N)+L(N) \\
& \leqq\left(2^{n-1}-1\right)+1+\left(2^{n-1}-1\right)=2^{n}-1 .
\end{aligned}
$$

The case $n=1$ being straightforward from the Wedderburn-Artin 
Theorem, the proof is complete.

Next we show that, even though the preceding theorem severely limits the possible disparity between $L\left(R_{R}\right)$ and $L\left({ }_{R} R\right)$ for a ring $R$ of finite Loewy length, the two lengths can be different. To do so, we employ the following variation of the standard construction used in adjoining an identity to a ring.

Lemma 1.4. Let $S$ be an ideal in a ring $T$. Let $K$ be a subring of T. Let $\varphi:{ }_{K} S_{T} \rightarrow{ }_{K} S_{T}$ be a bimodule homomorphism with kernel $N$. Let $R=K \times S$, and define addition in $R$ coordinatewise and multiplication by

$$
(k, s)\left(k^{\prime}, s^{\prime}\right)=\left(k k^{\prime}, s k^{\prime}+k s^{\prime}+s \varphi\left(s^{\prime}\right)\right) .
$$

Then $R$ is a ring with identity $(1,0)$ and $0 \times S$ and $0 \times N$ are ideals in $R$ with $(0 \times S)(0 \times N)=0$.

Proof. The fact that $R$ is a ring is a simple calculation. Note that if $\varphi$ is the identity map, one is simply adjoining a unit to $S$, and if not, the linearity of $\varphi$ allows us to move the $\varphi$ through a product to preserve associativity. The fact that $(0 \times S)(0 \times N)=0$ is straightforward.

Note that, in order to be of minimal Loewy length, the counterexample that we are seeking must have nonzero radical, because in a ring $R$ with zero radical $\operatorname{Soc}\left({ }_{R} R\right)=\operatorname{Soc}\left(R_{R}\right)$. However, an application of (1.1) to our following example will yield a Loewy ring with zero radical and differing finite Loewy lengths.

EXAMPLE 1.5. A Loewy ring $R$ with $L\left(R_{R}\right)=2$ and $L\left({ }_{R} R\right)=3$.

Demonstration. Let $T$ be the ring of linear transformations End $\left(V_{K}\right)$ of an infinite dimensional vector space over a field $K$. Let $S=\operatorname{Soc}(T)$. Then $S_{T}$ is an infinite direct sum of isomorphic minimal right ideals and so there is an epimorphism

$$
\varphi:{ }_{K} S_{T} \longrightarrow{ }_{K} S_{T} \text { with } \quad N=\operatorname{Ker} \varphi \neq 0 .
$$

Let $R=K \times S$ be the ring constructed from this data via Lemma 1.4. Then since $(0, s)\left(k^{\prime}, s^{\prime}\right)=\left(0, s\left(k^{\prime}+\varphi\left(s^{\prime}\right)\right)\right), \varphi$ is epic, and $M S=M$ for every $M_{T} \leqq S_{T}$, the right $R$-submodules of $0 \times S$ are just those of the form $0 \times M$ where $M \leqq S_{T}$. Thus $0 \times S$ is a non-artinian semisimple right ideal in $R$ and so, since $R /(0 \times S) \cong K$, we have $L\left(R_{R}\right)=2$. Now to see that $L\left({ }_{R} R\right)=3$, first note that, since by (1.4) $(0 \times S)(0 \times N)=0$, the left $R$-action on $0 \times N$ is just that of $K$. 
So $0 \times N$ is left semisimple over $R$. Moreover, being a nonzero right ideal in the $P M I$ ring $T, N_{T}$ is faithful. So if $k \neq 0$ or $s \notin N$ then there is an $n \in N$ with

$$
(0, n)(k, s)=(0, n(k+\varphi(s))) \neq 0
$$

because no transformation of finite rank can be scalar unless it is zero. Thus it follows that $0 \times N$ is essential in ${ }_{R} R$. Hence $0 \times N=$ Soc $\left({ }_{R} R\right)$. But $0 \times N \neq 0$ and clearly $R /(0 \times N)$ is not artinian, so by (1.3) we do have $L\left({ }_{R} R\right)=3$.

Note that, according to the inequality of (1.3), $L\left({ }_{R} R\right)=3$ is the largest possible for a Loewy ring with $L\left(R_{R}\right)=2$. We do not know whether the bound $2^{n}-1$ on $L\left({ }_{R} R\right)$ (given $L\left(R_{R}\right)=n$ ) can be achieved for any $n>2$.

2. Regular Loewy rings. In [6] Năstăsescu and Popescu proved that a commutative Loewy ring with zero radical is (von Neumann) regular. Subsequently, Năstăsescu [5] proved that a right Loewy ring is regular iff $J\left(R / S_{\alpha}\right)=0$ for each $\alpha<L\left(R_{R}\right)$. Since the left and right socles of a ring with zero radical are equal [3, Theorem 1, p. 65], the left and right Loewy series for such a ring must be identical. Our concluding results show how to construct both commutative and primitive regular Loewy rings of any length. Unlike our first example there is, of course, no $T$-nilpotence involved. The basic construction is based upon the following lemma about partitions of an uncountable set.

LEMMA 2.1. Let $\beta$ be an ordinal, $c$ be an infinite cardinal and let $A$ be a set such that card $A>c \geqq$ card $\beta$. Then there is a sequence of partitions $\left\{\mathscr{P}^{\alpha} \mid \alpha \leqq \beta\right\}$ of the set $A$ such that:

(1) Whenever $\alpha_{1}<\alpha_{2}, \mathscr{P}^{\alpha_{1}}$ is a refinement of $\mathscr{P}^{\alpha_{2}}$ but no member of $\mathscr{P}^{\alpha_{2}}$ is a finite union of members of $\mathscr{P}^{\alpha_{1}}$.

(2) Each element of $\mathscr{P}^{\alpha}$ has cardinality $\leqq c$ and card $\mathscr{P}^{\alpha}=$ card $A$ for all $\alpha$.

Proof. Let $\mathscr{P}^{0}$ be the discrete partition of $A$. If $a \in A$ let $P_{a}^{\alpha}$ be the member of $\mathscr{P}^{\alpha}$ containing $a$. The proof is by induction.

First, suppose $\alpha$ is a successor. Then, since card $\mathscr{P}^{\alpha-1}=\operatorname{card} A$ and $c \leqq$ card $A$, we can write $\mathscr{P}^{\alpha-1}$ as a disjoint union of card $A$ sets each of cardinality $c$. If $X$ is an element of the partition of $\mathscr{P}^{\alpha-1}$ described in the previous sentence, we take $Q(X)=\mathrm{U}_{X} P_{a}^{\alpha-1}$. The set of all such $Q(X)$ is a partition of $A$, clearly having the desired properties. 
If $\alpha$ is a limit ordinal, we define $P_{a}^{\alpha}=\bigcup_{\alpha^{\prime}<\alpha} P_{a}^{\alpha^{\prime}}$ and let $\mathscr{P}^{\alpha}=$ $\left\{P_{a}^{\alpha}\right\}$. First, $\mathscr{P}^{\alpha}$ is a partition. For suppose $P_{a}^{\alpha} \cap P_{b}^{\alpha} \neq \varnothing$. Then there are ordinals $\alpha_{1} \leqq \alpha_{2}<\alpha$ with $P_{a}^{\alpha_{1}} \cap P_{b}^{\alpha_{2}} \neq \varnothing$. But since $\mathscr{P}^{\alpha_{1}}$ is a refinement of $\mathscr{P}^{\alpha_{2}}$, we have $P_{a}^{\alpha_{1}} \subset P_{a}^{\alpha_{2}}$, so $P_{a}^{\alpha_{2}} \cap P_{b}^{\alpha_{2}} \neq \varnothing$. Thus, $P_{a}^{\alpha_{2}}=P_{b}^{\alpha_{2}}$. Now, if $y \in P_{a}^{\alpha}$, then $y \in P_{a}^{\delta}$, where $\delta<\alpha$. Thus, $y \in P_{a}^{\delta^{\prime}}$ for all $\delta^{\prime} \geqq \delta$. Thus, we may assume $\delta \geqq \alpha_{2}$. But since $P_{a}^{\alpha_{2}}=P_{b}^{\alpha_{2}}$, $P_{a}^{\delta}=P_{b}^{\delta}$ so that $y \in P_{b}^{\delta} \subset P_{b}^{\alpha}$, and, since $y$ was arbitrary $P_{a}^{\alpha} \subset P_{b}^{\alpha}$, and since the argument is symmetric, the $P_{a}^{a}$ are pairwise disjoint. Thus, since clearly $A=\bigcup_{a \in A} P_{a}^{\alpha}$, we have partitions $\left\{\mathscr{P}^{\alpha} \mid \alpha \leqq \beta\right\}$. For these part (1) is clear by construction, and (2) also follows for successor ordinals by construction. If, however, $\alpha$ is a limit ordinal then $P_{a}^{a}=\bigcup_{\delta<\alpha} P_{a}^{o}$. So card $P_{a}^{\alpha} \leqq \sup _{\delta<\alpha}\left(\operatorname{card} P_{a}^{\delta}\right)(\operatorname{card} \alpha)$. But card $P_{a}^{\delta} \leqq c$ by induction, and card $\alpha \leqq c$ so card $P_{a}^{\alpha} \leqq c^{2}=c$. Now, card $A \leqq$ $\left(\operatorname{card} \mathscr{P}^{\alpha}\right)\left(\sup _{a \in A}\right.$ card $\left.P_{a}^{\alpha}\right)$. But card $P_{a}^{\alpha} \leqq c$, so card $A \leqq\left(\operatorname{card} \mathscr{P}^{\alpha}\right) \cdot c$, while $c<\operatorname{card} A$. So, card $A \leqq \operatorname{card} \mathscr{P}^{\alpha}$ but $\mathscr{P}^{\alpha}$ is a partition of $A$ so card $\mathscr{P}^{\alpha} \leqq$ card $A$, and we have equality.

THEOREM 2.2. Let $\beta$ be any ordinal, then there is a commutative von Neumann regular ring $R_{\beta}$ such that every $R_{\beta}$ module has nontrivial socle, and $R_{\beta}$ has Loewy length $\beta+1$.

Proof. Let $A$ be a set with $\operatorname{card} A>\operatorname{card} \beta$ and let $\left\{\mathscr{P}^{\alpha} \mid \alpha \leqq \beta\right\}$ be the partitions of Lemma 2.1. Let $K$ be a field and let $T=K^{A}$, the ring direct product of card $A$ copies of $K$ with projections $\pi_{a}: T \rightarrow K(a \in A)$. For each ordinal $\alpha<\beta$ and each $P_{a}^{\alpha} \in \mathscr{P}^{\alpha}$ define $\mathfrak{w}\left(P_{a}^{a}\right) \in T$

$$
\pi_{b}\left(\mathfrak{w}\left(P_{u}^{\alpha}\right)\right)=1 \quad \text { if } \quad b \in P_{a}^{\alpha}
$$

and

$$
\pi_{b}\left(\mathfrak{w}\left(P_{a}^{a}\right)\right)=0 \quad \text { if } \quad b \in A \backslash P_{a}^{\alpha}
$$

and let $1_{T}$ be the identity of $T$. Then from (2.1.1) it is easy to see that

$$
\left\{1_{T}\right\} \cup\left\{\mathfrak{w}\left(P_{a}^{\alpha}\right) \mid \alpha<\beta, P_{a}^{\alpha} \in \mathscr{P}_{\alpha}\right\}
$$

is a linearly independent set of vectors in $T_{K}$. Let $R_{\beta}$ be the subspace that it spans. We shall prove that $R_{\beta}$ is a subalgebra of $T$ with the desired properties. The proof is facilitated by the following observation: If $\alpha_{1} \leqq \alpha_{2}$, then

$$
\mathfrak{w}\left(P_{a}^{\alpha_{1}}\right)\left(\mathfrak{w} P^{\alpha_{2}}\right)=\left\{\begin{array}{cl}
\mathfrak{w}\left(P_{a}^{\alpha_{1}}\right), & \text { if } P_{a}^{\alpha_{1}} \leqq P_{b}^{a_{2}} \\
0, & \text { otherwise } .
\end{array}\right.
$$

This is true because $\mathscr{P}^{\alpha_{1}}$ is a refinement of $\mathscr{P}^{a_{2}}$ so that if $P_{a}^{\alpha_{1}} \nsubseteq P_{b}^{\alpha_{2}}$ then $P_{a}^{\alpha_{1}} \cap P_{b}^{\alpha_{2}}=\varnothing$. It shows immediately that $R_{\beta}$ is a subalgebra of T. Now for each $\alpha<\beta$, let 


$$
L_{\alpha}=\sum_{\mathscr{Q} \alpha} \mathfrak{w}\left(P_{a}^{\alpha}\right) K
$$

and for each $\alpha \leqq \beta$ set

$$
S_{\alpha}=\sum_{\delta<\alpha} L_{\delta}
$$

(so $S_{0}=0$ ). Then from $(*)$ it also follows that

$$
S_{\alpha}(\alpha \leqq \beta) \text { and } S_{\alpha}+\mathfrak{w}\left(P_{a}^{a}\right) K(\alpha<\beta)
$$

are ideals of $R_{\beta}$. But as $K$-spaces

$$
S_{\alpha+1}=S_{\alpha} \oplus L_{\alpha}=S_{\alpha} \oplus\left(\bigoplus_{\mathscr{\alpha} \alpha} \mathfrak{w}\left(P_{a}^{\alpha}\right) K\right),
$$

for each $\alpha<\beta$. Thus $S_{\alpha+1} / S_{\alpha}$ is a direct sum

$$
S_{\alpha+1} / S_{\alpha}=\bigoplus_{\text {⿻ } \alpha}\left(\mathfrak{m}\left(P_{a}^{a}\right) K+S_{\alpha}\right) / S_{\alpha}
$$

of minimal ideals of $R / S_{\alpha}$. Therefore, $R_{\beta}=S_{\beta}+1_{T} K$ is a Loewy ring; and to see that it has the desired length we need only show that $S_{\alpha+1} / S_{\alpha}$ is essential in $R / S_{\alpha}$ for each $\alpha<\beta$. So let $\alpha<\beta$ and

$$
r=\sum_{i=1}^{n} \mathfrak{m}\left(P_{a_{i}}^{a_{i}}\right) k_{i} \in R \backslash S_{\alpha}
$$

with the $k_{i} \neq 0$, the $P_{a_{i}}^{\alpha_{i}}$ distinct members of $\bigcup_{\tilde{\delta}<\beta} \mathscr{P}^{\dot{\delta}}$ and

$$
\alpha_{1} \leqq \cdots \leqq \alpha_{k-1}<\alpha \leqq \alpha_{k+1} \leqq \cdots \leqq \alpha_{n} .
$$

Then by (2.1.1) $P_{a_{n}}^{\alpha_{n}} \neq \bigcup_{i=1}^{n-1} P_{a_{i}}^{a_{i}}$, but $P_{a_{n}}^{\alpha_{n}}$ is a union of members of $\mathscr{P}$. So there exists a $P_{a}^{\alpha} \in \mathscr{P}^{\alpha}$ such that $P_{a}^{\alpha} \cong P_{a_{n}}^{\alpha_{n}}$ but $P_{a}^{\alpha} \nsubseteq P_{a_{2}}^{\alpha_{i}}(i=$ $1, \cdots, n-1)$. Now by $(*)$ we have

$$
r \mathfrak{w}\left(P_{a}^{\alpha}\right)=\sum_{i=1}^{k-1} \mathfrak{w}\left(P_{a_{i}}^{\alpha_{i}}\right) \mathfrak{w}\left(P_{a}^{\alpha}\right) k_{i}+\mathfrak{w}\left(P_{a}^{\alpha}\right) k_{n} \in S_{\alpha+1} \mid S_{\alpha},
$$

which shows that $S_{\alpha+1} / S_{\alpha}$ is indeed essential in $R_{\beta} / S_{\alpha}$. Thus $L\left(R_{\beta}\right)=$ $\beta+1$. Finally, since $R_{\beta}$ is commutative, to see that it is regular we need only, by [6, Theorem 3.1], observe that $J\left(R_{\beta}\right)=0$. But this is the case because each $\mathfrak{w}\left(P_{a}^{a}\right)$ is an idempotent in $R_{\beta}$.

COROLLARY 2.3. There are primitive regular Loewy rings of Loewy length $\beta+1$ for each ordinal $\beta$.

Proof. Use the ring $R_{\beta}$ and Lemma 1.1 to construct a $P M I$ ring $R=\hat{R}_{\beta}+S$ with

$$
\operatorname{Soc}(R)=S, \quad \hat{R}_{\beta} \cap S=0 \text { and } \widetilde{R}_{\beta} \cong R_{\beta} ;
$$

and observe that $R$ has zero radical modulo each term in its Loewy series. Now apply the result of Năstăsescu [5] cited above. 
3. Remarks. (1) Since minimal one-sided ideals are either nilpotent or idempotent generated, semiprime (left or right) Loewy rings have zero Jacobson radical.

( 2) Over a direct product of rings $\Pi_{A} R_{\alpha}$, the factor $\Pi_{A} R_{\alpha} / \oplus_{A} R_{\alpha}$ contains no minimal one-sided ideals. Thus no infinite direct product of rings is a Loewy ring.

(3) Let $R$ be a left or right self-injective right Loewy ring with $J(R)=0$. Then $R$ is a direct product of endomorphism rings of vector spaces (see [2]). By (2) this product must be finite. But also the vector spaces must be finite dimensional (for otherwise $R$ would have a non-Loewy factor ring). Thus $R$ is a semisimple ring.

(4) Left perfect (= right Loewy with $R / J(R)$ semisimple) rings have the property that each of their nonzero left modules contains a maximal submodule. So do commutative Loewy rings (see [6] and [4]) and right Loewy rings of finite length (see [6], Proposition 5.3], and our Theorem 1.3). We suspect that every right Loewy ring has this property.

We wish to express our appreciation to Professor Goro Azumaya. It was a recent conversation with him that stimulated our interest in this subject. We also wish to thank Professor John Beachy for guiding us to the appropriate literature.

Added in proof. T. Shores has kindly informed us that L. Fuchs had obtained Theorem 2.2 earlier in Theorem 6 of [J. Reine Angew. Math. 239/240 (1970), 169-179].

\section{REFERENCES}

1. H. Bass, Finitistic dimension and a homological generalization of semiprimary rings, Trans. Amer. Math. Soc., 95 (1960), 466-488.

2. S. U. Chase and C. C. Faith, Quotient rings and direct products of full linear rings, Math. Z., 88 (1965), 250-264.

3. N. Jacobson, Structure of Rings, Amer. Math. Soc. Coll. Pub., 1964, Providence, R. I.

4. L. A. Koifman, Rings over which every module has a maximal submodule, Math. Notes of the Acad. of Sci. of the USSR, vol. 7, no. 3, (Mar.-April 1970).

5. C. Năstăsescu, Quelques remarques sur une classe d'anneaux, C. R. Acad. Sci. Paris, 270 (1 April, 1970).

6. C. Năstăsescu and N. Popescu, Anneaux semi-artinians, Bull. Soc. Math. France, 96 (1968), 357-368.

7. B. L. Osofsky, Loewy length of perfect rings, Proc. Amer. Math. Soc., 20 (1971), 252-254.

Received June 28, 1973. The second author's research was supported by NSF Grant, GP-18828. 


\section{PACIFIC JOURNAL OF MATHEMATICS}

\section{EDITORS}

RICHARD ARENS (Managing Editor)

University of California

Los Angeles, California 90024

\section{J. DUGUNDJI}

Department of Mathematics University of Southern California Los Angeles, California 90007

D. Gilbarg and J. Milgram

Stanford University

Stanford, California 94305

University of Washington
Seattle, Washington 98105

ASSOCIATE EDITORS
E. F, BECKENBACH
B. H. NEUMANN
F. WOLF
K. Yoshida

\section{SUPPORTING INSTITUTIONS}

\author{
UNIVERSITY OF BRITISH COLUMBIA \\ CALIFORNIA INSTITUTE OF TECHNOLOGY \\ UNIVERSITY OF CALIFORNIA \\ MONTANA STATE UNIVERSITY \\ UNIVERSITY OF NEVADA \\ NEW MEXICO STATE UNIVERSITY \\ OREGON STATE UNIVERSITY \\ UNIVERSITY OF OREGON \\ OSAKA UNIVERSITY
}

\author{
UNIVERSITY OF SOUTHERN CALIFORNIA \\ STANFORD UNIVERSITY \\ UNIVERSITY OF TOKYO \\ UNIVERSITY OF UTAH \\ WASHINGTON STATE UNIVERSITY \\ UNIVERSITY OF WASHINGTON \\ * * * * \\ AMERICAN MATHEMATICAL SOCIETY \\ NAVAL WEAPONS CENTER
}

The Supporting Institutions listed above contribute to the cost of publication of this Journal, but they are not owners or publishers and have no responsibility for its content or policies.

Mathematical papers intended for publication in the Pacific Journal of Mathematics should be in typed form or offset-reproduced, (not dittoed), double spaced with large margins. Underline Greek letters in red, German in green, and script in blue. The first paragraph or two must be capable of being used separately as a synopsis of the entire paper. Items of the bibliography should not be cited there unless absolutely necessary, in which case they must be identified by author and Journal, rather than by item number. Manuscripts, in duplicate if possible, may be sent to any one of the four editors. Please classify according to the scheme of Math. Rev. Index to Vol. 39. All other communications to the editors should be addressed to the managing editor, or Elaine Barth, University of California, Los Angeles, California, 90024.

100 reprints are provided free for each article, only if page charges have been substantially paid. Additional copies may be obtained at cost in multiples of 50 .

The Pacific of Journal Mathematics is issued monthly as of January 1966. Regular subscription rate: $\$ 72.00$ a year (6 Vols., 12 issues). Special rate: $\$ 36.00$ a year to individual members of supporting institutions.

Subscriptions, orders for back numbers, and changes of address should be sent to Pacific Journal of Mathematics, 103 Highland Boulevard, Berkeley, California, 94708.

PUBLISHED BY PACIFIC JOURNAL OF MATHEMATICS, A NON-PROFIT CORPORATION

Printed at Kokusai Bunken Insatsusha (International Academic Printing Co., Ltd.), 270, 3-chome Totsuka-cho, Shinjuku-ku, Tokyo 160, Japan.

Copyright (C) 1973 by Pacific Journal of Mathematics Manufactured and first issued in Japan 


\section{Pacific Journal of Mathematics}

\section{Vol. 53, No. $2 \quad$ April, 1974}

Kenneth Abernethy, On characterizing certain classses of first countable spaces by

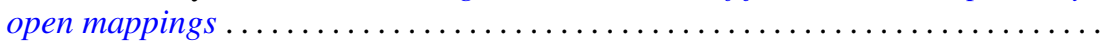

Ross A. Beaumont and Donald Lawver, Strongly semisimple abelian groups .......

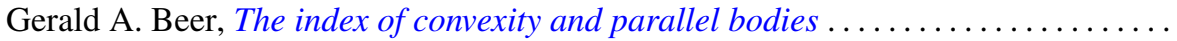

Victor P. Camillo and Kent Ralph Fuller, On Loewy length of rings ..............

Stephen LaVern Campbell, Linear operators for which $T^{*} T$ and $T T^{*}$ commute.

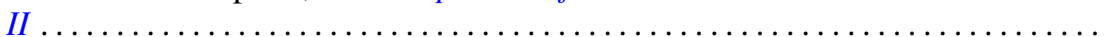

Charles Kam-Tai Chui and Philip Wesley Smith, Characterization of a function by

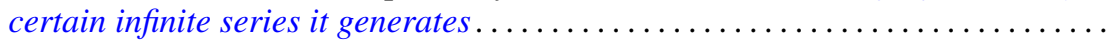

Allan L. Edelson, Conjugations on stably almost complex manifolds . ...........

Patrick John Fleury, Hollow modules and local endomorphism rings . . ..........

Jack Tilden Goodykoontz, Jr., Connectedness im kleinen and local connectedness in

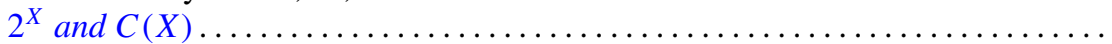

Robert Edward Jamison, II, Functional representation of algebraic intervals .......

Athanassios G. Kartsatos, Nonzero solutions to boundary value problems for

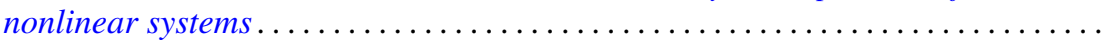

Soon-Kyu Kim, Dennis McGavran and Jingyal Pak, Torus group actions on simply

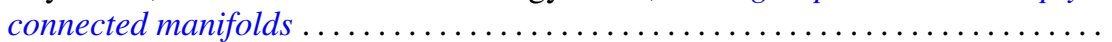

David Anthony Klarner and R. Rado, Arithmetic properties of certain recursively

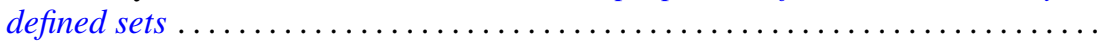

Ray Alden Kunze, On the Frobenius reciprocity theorem for square-integrable

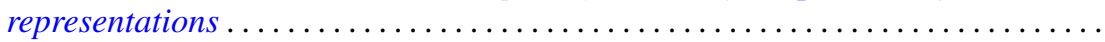

John Lagnese, Existence, uniqueness and limiting behavior of solutions of a class of differential equations in Banach space...

Teck Cheong Lim, A fixed point theorem for families on nonexpansive mappings Lewis Lum, A quasi order characterization of smooth continua

Andy R. Magid, Principal homogeneous spaces and Galois extensions . .

Charles Alan McCarthy, The norm of a certain derivation ..... . .

Louise Elizabeth Moser, On the impossibility of obtaining $S^{2} \times S^{1}$ by elementary surgery along a knot. .

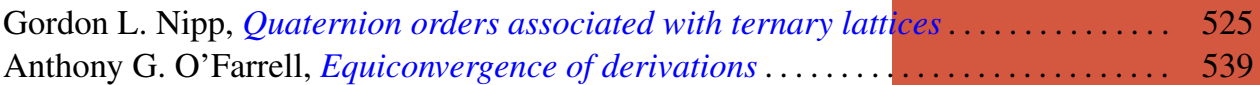

Dorte Olesen, Derivations of $A W^{*}$-algebras are inner . . . . . . . . . . . . . . . 555

Dorte Olesen and Gert Kjærgaard Pedersen, Derivations of $C^{*}$-algebras have

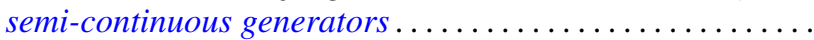

Duane O’Neill, On conjugation cobordism.

Chull Park and S. R. Paranjape, Probabilities of Wiener paths crossing differentiable

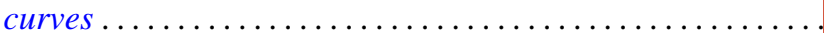

Edward Ralph Rozema, Almost Chebyshev subspaces of $L^{1}(\mu$;

Lesley Millman Sibner and Robert Jules Sibner, A note on the Atiyah-Bott fixed

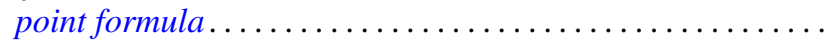

Betty Salzberg Stark, Irreducible subgroups of orthogonal groups generated by

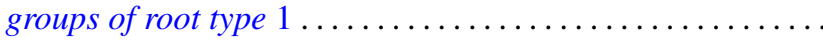

N. Stavrakas, A note on starshaped sets, $(k)$-extreme points and the half ray

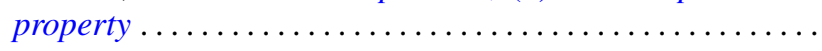

Carl E. Swenson, Direct sum subset decompositions of $Z \ldots \ldots$ 